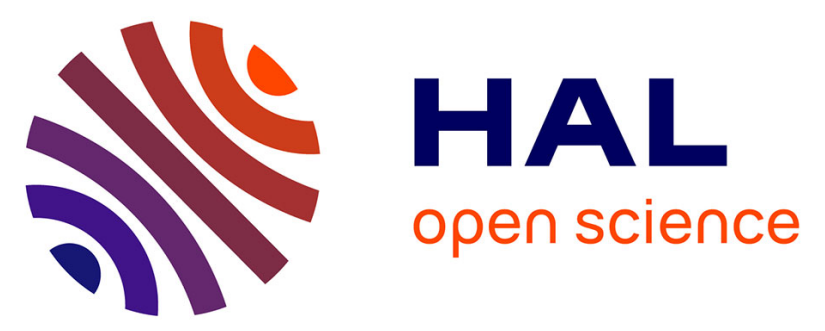

\title{
Crizotinib : enfin l'AMM en 1re ligne des cancers bronchiques non à petites cellules de stade avancé ALK+
}

\author{
Etienne Giroux Leprieur, Marie Wislez
}

\section{To cite this version:}

Etienne Giroux Leprieur, Marie Wislez. Crizotinib : enfin l'AMM en 1re ligne des cancers bronchiques non à petites cellules de stade avancé ALK+. Bulletin du Cancer, 2016, 103 (2), pp.125-126. 10.1016/j.bulcan.2015.12.002 . hal-01299745

\section{HAL Id: hal-01299745 \\ https://hal.sorbonne-universite.fr/hal-01299745}

Submitted on 8 Apr 2016

HAL is a multi-disciplinary open access archive for the deposit and dissemination of scientific research documents, whether they are published or not. The documents may come from teaching and research institutions in France or abroad, or from public or private research centers.
L'archive ouverte pluridisciplinaire HAL, est destinée au dépôt et à la diffusion de documents scientifiques de niveau recherche, publiés ou non, émanant des établissements d'enseignement et de recherche français ou étrangers, des laboratoires publics ou privés. 
Crizotinib : enfin l'AMM en $1^{\text {ière }}$ ligne des cancers bronchiques non à petites cellules de stade avancé ALK+

Crizotinib: at last in first-line treatment of advanced-stage ALK-rearranged non-small cell lung cancer

Etienne Giroux Leprieur ${ }^{1,2}$, Marie Wislez ${ }^{3,4}$

1- Service de Pneumologie et Oncologie thoracique, APHP - Hôpital Ambroise Paré, 9 avenue Charles de Gaulle, F-92100 Boulogne-Billancourt

2- Laboratoire EA4340, Biomarqueurs en Cancérologie et Onco-Hématologie, Université Versailles - Saint Quentin en Yvelines, 9 avenue Charles de Gaulle, F-92100 Boulogne-Billancourt

3- Service de Pneumologie, APHP - Hôpital Tenon, 4 rue de la Chine, F-75020 Paris

4- Sorbonne Universités, UPMC Univ Paris 06, GRC n04, Theranoscan, F-75252, Paris

Auteur correspondant : Pr Marie Wislez, Service de Pneumologie, APHP - Hôpital Tenon, 4 rue de la Chine, 75020 Paris. Tel: +33 (0)156016838. Fax: +33 (0)156017248. Email: marie.wislez@tnn.aphp.fr

Mots-clés : cancer bronchique non à petites cellules ; ALK ; crizotinib ; inhibiteur de tyrosine kinase 
La présence d'un réarrangement de $A L K$ concerne environ $4 \%$ des cancers bronchiques non à petites cellules (CBNPC). Il induit une sensibilité des cellules tumorales aux inhibiteurs de tyrosine kinase (ITK) de ALK, tel que le crizotinib. L'essai de phase I publié en 2010 a testé le crizotinib (250mg x2/jour) en $2^{\text {ième }}$ ligne et plus chez 82 patients ayant un CBNPC de stade avancé avec un réarrangement de $A L K$ (1). Le taux de réponse était de $57 \%$, avec un profil de toxicité acceptable. Des données actualisées (2) sur une extension de cohorte de 143 patients publiées en 2012 montraient un taux de réponse de 60,8\% et une survie sans progression (SSP) de 9,7 mois. Un essai de phase II (PROFILE-1005) a montré un taux de réponse de 59,8\% et une SSP de 8,1 mois chez 261 patients (3). L'essai de phase III PROFILE-1007, publié en 2013, a comparé, toujours en $2^{\text {ième }}$ ligne et plus, le crizotinib à une chimiothérapie par pemetrexed ou docetaxel (4). Le taux de réponse était de $65 \%$ (versus $20 \%$ avec chimiothérapie, $\mathrm{p}<0,0001$ ) et la SSP de 7,7 mois (versus 3 mois avec chimiothérapie, $\mathrm{p}<0,0001$ ). Ces résultats ont permis au crizotinib (Xalkori ${ }^{\mathrm{TM}}$, Pfizer) d'obtenir aux Etats-Unis l'autorisation de la Food and Drug Administration (FDA) en 2011, et en France 1'Autorisation de Mise sur le Marché (AMM) en 2012 en $2^{\text {ième }}$ ligne et plus chez des CBNPC de stade avancé avec réarrangement de $A L K$, à la posologie de $250 \mathrm{mg}$ x2/jour (5). Plus récemment, l'essai de phase III PROFILE-1014 a comparé le crizotinib cette fois-ci en en 1 ière ligne à un doublet à base de platine (6). Cet essai a inclus 343 patients, randomisés entre un bras crizotinib (250 mg x2/jour) et un bras chimiothérapie (pemetrexed $500 \mathrm{mg} / \mathrm{m}^{2}$ et cisplatine $75 \mathrm{mg} / \mathrm{m}^{2}$ ou carboplatine AUC 5 ou 6) toutes les 3 semaines pour 6 cycles. La prescription de crizotinib en $2^{\text {ième }}$ ligne à la progression dans le bras chimiothérapie était autorisée. Le taux de réponse était de $74 \%$ avec le crizotinib (versus $45 \%$ avec chimiothérapie, $\mathrm{p}<0,0001$ ), et la SSP (objectif principal) de 10,9 mois (versus 7,0 mois avec chimiothérapie; p<0,0001). La survie à 1 an était similaire, $84 \%$ dans le bras crizotinib, versus $79 \%$ dans le bras chimiothérapie, probablement du fait du cross-over entre les bras de 
traitement. Les effets secondaires les plus fréquents du crizotinib étaient les troubles visuels (71\%), la diarrhée (61\%), les œedèmes (49\%), les vomissements $(46 \%)$ et la constipation (43\%). Les effets secondaires de grade 3-4 les plus fréquents du crizotinib étaient la cytolyse (14\%) et la neutropénie (11\%). Le taux d'arrêt de traitement pour toxicité était équivalent dans les 2 bras (12\% crizotinib et 14\% chimiothérapie). La qualité de vie était plus améliorée avec le crizotinib qu'avec la chimiothérapie. On notera aussi que le bras contrôle était non optimal, sans bevacizumab ni maintenance de continuation par pemetrexed. Le cross-over à la progression ne peut pas non plus permettre de dégager un éventuel bénéfice de survie globale du crizotinib. Sur la base de ces résultats, le Comité des Médicaments à usage Humain (Committee for Medicinal Products for Human Use, CHMP) de l'Agence Européenne des Médicaments (EMA) a recommandé une extension d'indication du crizotinib dès la $1^{\text {ière }}$ ligne pour les CBNPC avec réarrangement de $A L K$ le 23 novembre 2015. Contrairement aux ITK de $1^{\prime} E G F R$ où l'efficacité est équivalente en $1^{\text {ière }}$ ou $2^{\text {ième }}$ ligne, le taux de réponse avec le crizotinib apparaît ici supérieur en $1^{\text {ière }}$ ligne par rapport à son utilisation en $2^{\text {ième }}$ ligne ou plus (74\% versus $60,8 \%$, respectivement), tout comme la SSP (10,9 mois dans PROFILE-1014 et 7,7 mois dans PROFILE-1007), ce qui est un argument supplémentaire pour un screening rapide du statut $A L K$ dès le diagnostic.

Notre nouveau défi est maintenant la gestion de la progression sous crizotinib. Plusieurs mécanismes sont maintenant décrits (7); dans un tiers des cas, il s'agit de mécanismes ALK dépendants : amplification du gène $A L K$, apparition d'une mutation de résistance au niveau du domaine tyrosine kinase ; dans un tiers des cas, le mécanisme ALK indépendant: acquisition d'une mutation d'EGFR, Kras, activation de la voie ErbB, IGFR ou c-Kit, transition épithélio-mésenchymateuse, autophagie et dans un tiers des cas, aucun mécanisme n’est mis en évidence. Ces mécanismes de résistance ont été décrits sur de très petits effectifs de patients, et de plus grandes cohortes sont nécessaires pour mieux préciser la fréquence 
respective de ces anomalies. De nouveaux ITK de ALK, dits de $2^{\text {ième }}$ génération, ont été ou sont en cours de développement. C'est le cas du ceritinib, qui a l'AMM depuis cette année dans les CBNPC de stade avancé avec réarrangement de $A L K$ après échec ou intolérance au crizotinib $(8,9)$. L'alectinib semble efficace dans ces mêmes indications (10). Apres échec du crizotinib, les taux de réponse sont élevés, $58 \%$ pour le ceritinib (8) et $50 \%$ avec l'alectinib (10), y compris au niveau cérébral. D'autres médicaments sont en développement tels le lorlatinib. Comme pour les ITK de l'EGFR de $3^{\text {ième }}$ génération, se pose la question de la stratégie d'utilisation des ITK de ALK. Faut-il utiliser le crizotinib en $1^{\text {ière }}$ ligne et réserver les ITK de $2^{\text {ième }}$ génération en $2^{\text {ième }}$ intention, où faut-il utiliser d'emblée l'alectinib ou le ceritinib ? Ce sont probablement les différences d'activité des ITK de $2^{\text {ième }}$ génération sur les différents spectres des mutations de résistance acquise qui dictera le choix de la molécule (7). Ainsi, le ceritinib semble actif en cas de mutation $A L K$ I1171T ou V1180L décrit après alectinib. A l'inverse, l'alectinib est efficace en cas de mutation G1123S, décrit après ceritinib (7). Comme pour les CBNPC mutés EGFR, il semble donc important de documenter le mécanisme de progression sous crizotinib et autre ITK de ALK afin de guider le choix des différentes possibilités actuelles. Il reste néanmoins une place indéniable pour la chimiothérapie conventionnelle - avec une sensibilité particulière des patients $A L K+$ au pemetrexed (11) - et dont la séquence optimale reste à définir.

1. Kwak EL, Bang YJ, Camidge DR, Shaw AT, Solomon B, et al. Anaplastic lymphoma kinase inhibition in non-small-cell lung cancer. N Engl J Med 2010;363:1693-703.

2. Camidge DR, Bang YJ, Kwak EL, Iafrate AJ, Varella-Garcia M, et al. Activity and safety of crizotinib in patients with ALK-positive non-small-cell lung cancer: updated results from a phase 1 study. Lancet Oncol 2012;13:1011-9. 
3. Kim D, Ahn M, Yang P, Liu X, De Pas T, et al. Updated results of a global phase II study with Crizotinib in advanced ALK-positive non-small cell lung cancer (NSCLC). Ann Oncol 2012;23(suppl 9):abstr1230PD.

4. Shaw AT, Kim DW, Nakagawa K, Seto T, Crinó L, et al. Crizotinib versus chemotherapy in advanced ALK-positive lung cancer. N Engl J Med 2013;368:2385-94.

5. Fallet V, Toper C, Antoine M, Cadranel J, Wislez M. Crizotinib, modalités pratiques d'un traitement personnalisé. Bull Cancer 2012;99:787-91.

6. Solomon BJ, Mok T, Kim DW, Wu YL, Nakagawa K, et al. First-line crizotinib versus chemotherapy in ALK-positive lung cancer. N Engl J Med 2014;371:2167-77.

7. Toyokawa G, Seto T. Updated Evidence on the Mechanisms of Resistance to ALK Inhibitors and Strategies to Overcome Such Resistance: Clinical and Preclinical Data. Oncol Res Treat 2015;38:291-8.

8. Shaw AT, Kim DW, Mehra R, Tan DSW, Felip E, et al. Ceritinib in ALK-rearranged non-small-cell lung cancer. N Engl J Med 2014;370:1189-97.

9. Giroux Leprieur E, Fallet V, Wislez M. Modalités d'utilisation du ceritinib (Zykadia ${ }^{\mathrm{TM}}$ ), inhibiteur de ALK de 2e génération, dans le cancer bronchique non à petites cellules de stade avancé. Bull Cancer 2015;102: 1053-1057.

10. Ou SHI, Ahn JS, De Petris L, Govindan R, Yang JCH, et al. Alectinib in CrizotinibRefractory ALK-Rearranged Non-Small-Cell Lung Cancer: A Phase II Global Study. J Clin Oncol 2015;doi: 10.1200/JCO.2015.63.9443. 
11. Park S, Park TS, Choi CM, Lee DH, Kim SW, et al. Survival Benefit of Pemetrexed in Lung Adenocarcinoma Patients With Anaplastic Lymphoma Kinase Gene Rearrangements. Clin Lung Cancer 2015;16:e83-9. 